\title{
Chemerin in a Mouse Model of Non-alcoholic Steatohepatitis and Hepatocarcinogenesis
}

\author{
ELISABETH M. HABERL ${ }^{1}$, REBEKKA POHL ${ }^{1}$, LISA REIN-FISCHBOECK ${ }^{1}$, \\ SUSANNE FEDER ${ }^{1}$, CHRISTOPHER J. SINAL $^{2}$ and CHRISTA BUECHLER ${ }^{1}$ \\ ${ }^{1}$ Department of Internal Medicine I, Regensburg University Hospital, Regensburg, Germany; \\ ${ }^{2}$ Department of Pharmacology, Dalhousie University, Halifax, Canada
}

\begin{abstract}
Background/Aim: Non-alcoholic steatohepatitis (NASH) is a risk factor for hepatocellular carcinoma (HCC). The adipokine chemerin protects from HCC and is reduced in human HCC. In this study, chemerin expression was analyzed in a murine model of NASH-HCC. Materials and Methods: Serum and hepatic chemerin, and ex vivo chemerin receptor activation were monitored in NASH and NASH-HCC in mice fed a low-methionine diet deficient in choline after initiation of tumors by injection of diethylnitrosamine. Results: In non-tumorous liver tissues, the extent of hepatic steatosis, and the levels of proteins regulating hepatic lipids and liver fibrosis were similar in NASH and NASH-associated HCC. Systemic and hepatic chemerin, and chemerin receptor activation were not changed in HCC. Liver tumors only developed in diethylnitrosamine-injected mice and their number was increased in NASH. Chemerin protein was induced in liver in NASH, but was unchanged in HCC tissues. Conclusion: Hepatic and serum chemerin and ex vivo analyzed chemerin receptor activation do not differ in murine NASH-associated HCC when compared to NASH. Hepatic tumors still develop despite high endogenous levels of serum and liver chemerin protein.
\end{abstract}

Metabolic disease in obesity is related to adipokine imbalance and low-grade chronic inflammation (1). The adipokine chemerin has a critical role in insulin response, adipogenesis and inflammation (2). Accordingly, total serum chemerin protein is elevated in murine and human obesity (3). Non-alcoholic fatty liver disease (NAFLD) is a common diagnosis in obesity and its progressive form, non-alcoholic steatohepatitis (NASH), promotes liver cirrhosis and carcinogenesis (1). Hepatocellular carcinoma (HCC) not only

Correspondence to: Christa Buechler, Ph.D., Franz-Josef Strauss Allee 11, 93042 Regensburg, Germany. Tel: +49 9419447009, e-mail: christa.buechler@klinik.uni-regensburg.de

Key Words: Liver tumor, NASH, chemerin activity, CMKLR1. develops in the cirrhotic liver but can also arise in the noncirrhotic liver in NASH (4). The molecular events involved in NASH progression and development of HCC are incompletely understood $(1,4)$. Chemerin is highly expressed in the liver and modulates insulin response and tumor growth, and may thus have a role therein (5-7).

Hepatic chemerin mRNA and protein expression have been analyzed in murine and human NASH (3). Liver chemerin protein is increased in murine NASH induced by feeding an atherogenic or a methionine-choline-deficient (MCD) diet (6). Elevated hepatic chemerin mRNA has been also described in human NAFLD (8). However, other studies reported unchanged or lower hepatic chemerin expression in human and murine NAFLD (3, 9-11).

Interestingly, a low level of chemerin in HCC tissues of patients has been associated with worse outcomes $(7,12)$. Correlation of chemerin protein levels with the number of dendritic and natural killer cells indicates a role for tumorresident chemerin in immune cell infiltration (12). In a preclinical study, chemerin-null mice exhibited more aggressive disease in a murine HCC model in which Hepa16 cells were implanted, and accordingly, tumor growth was reduced upon chemerin overexpression in Hepa1-6 cells. Furthermore, the growth of subcutaneous transplanted Hepa1-6 tumors was blocked by injection of recombinant chemerin. Chemerin also reduced angiogenesis in tumor tissues in this model, impaired the function of myeloidderived suppressor cells and enhanced the activity of antitumor T-cells (7).

The serum level of chemerin is further reported to be low in patients with HCC (13). In contrast to hepatic chemerin protein, levels of circulating chemerin are not correlated with disease recurrence or prognosis $(12,13)$. Chemerin is expressed in the liver and reduced production in this organ may contribute to low hepatic and serum levels in liver cirrhosis and eventually $\operatorname{HCC}(3,13)$. While one study did not report any effect of liver dysfunction on the systemic chemerin level, a further study described reduced serum chemerin in patients with decompensated liver cirrhosis (13, 
14). Thus, a low level of chemerin in patients with HCC may reflect hepatic dysfunction rather than carcinogenesis.

Chemerin is produced as an inactive precursor and is activated by extracellular proteases. Distinct C-terminally truncated isoforms are produced which activate the chemokine-like receptor 1 (CMKLR1) and G-proteincoupled receptor 1 (GPR1) with different efficiencies $(3,5$, 15). Chemerin induces the recruitment of $\beta$-arrestin 1 and 2 to both these receptors (15). Most analyses detect chemerin by enzyme-linked immunosorbent assays (ELISAs) (3, 5, 16). Yet variations in the total protein level do not provide information on the distribution of chemerin isoforms, and thus do not necessarily reflect changes in chemerin activity. Discrepancies between activated and total chemerin levels have indeed been described in lean and obese women (16) and murine models of obesity (17). Therefore a change in total chemerin level in patients with liver diseases (3) may not predict altered chemerin bioactivity.

NASH induced by an MCD diet in mice increases systemic and hepatic chemerin levels (6). An MCD diet promotes liver carcinogenesis and a high level of chemerin is purported to protect from this malignancy $(7,18)$. An MCD diet causes massive body weight loss making it difficult to maintain mice on this feed for the time necessary to develop liver cancer (19). A low-methionine-cholinedeficient (LMCD) diet induces NASH, but maintains body weight (20). In order to mimic the pathogenesis of NASHinduced HCC, the carcinogen diethylnitrosamine (DEN) can be used.

The aim of the present study was the comparative analysis of hepatic and serum chemerin levels in murine NASH and $\mathrm{NASH}$-associated liver cancer.

\section{Materials and Methods}

Animals. Male C3H/HeNRj mice were obtained from Janvier Labs (Le Genest-Saint-Isle, France). The 18- to 21-day-old mice were injected with $25 \mu \mathrm{g}$ DEN (Sigma, Taufkirchen, Germany)/g body weight (dissolved in water) to induce carcinogenesis. Injection of water served as a control. Mice were then fed a standard chow for 5 weeks. Subsequently, mice were fed either a control chow (E15668-04; Ssniff, Soest, Germany) or an LMCD diet (E1566794; Ssniff) for 16 weeks. The composition of the LMCD diet has been described by Toriguchi et al. (21). Animals had free access to food and water and were housed at $21 \pm 1^{\circ} \mathrm{C}$ under a $12 \mathrm{~h}$ light-dark cycle. Mice were killed at the age of 24 weeks under $\mathrm{CO}_{2}$-induced coma by cervical dislocation. Procedures were in accordance with the German Law on Animal Protection and the Institute for Laboratory Animal Research Guide for the Care and Use of Laboratory Animals from 1999 under approval number 54-2532.121/14. Experiments complied with the institutional and governmental regulations for animal use.

Quantification of total serum chemerin and adiponectin. ELISAs to measure murine chemerin and adiponectin were obtained from R\&D
Systems (Wiesbaden-Nordenstadt, Germany), and were performed as recommended by the distributor. Mouse serum was diluted 1,000fold for chemerin and 20,000-fold for adiponectin analysis. The sensitivity of the chemerin ELISA was $3.47 \mathrm{pg} / \mathrm{ml}$ and the range of the assay was $46.9-3,000 \mathrm{pg} / \mathrm{ml}$. The sensitivity of the adiponectin ELISA was $0.007 \mathrm{ng} / \mathrm{ml}$ and the range of the assay was $0.2-10 \mathrm{ng} / \mathrm{ml}$.

Measurement of systemic CMKLR1 and GPR1 activity. A HEK293 cell line (HTLA) that constitutively expresses a fusion protein consisting of a tobacco etch virus protease which is linked to murine $\beta$-arrestin 2 was used. The cells were transiently transfected with a plasmid encoding for murine CMKLR1 or GPR1 fused to a transcriptional trans-activator-dependent luciferase reporter gene via a tobacco etch virus protease recognition sequence. Details of the analysis of ex vivo chemerin receptor activation have been described elsewhere $(16,22)$. Recombinant chemerin-156 which is the most active isoform regarding $\beta$-arrestin 2 recruitment was purchased from $R \& D$ Systems and was diluted to $0.1,0.3,0.6,1.0,3.0,6.0,10.0$ and $30.0 \mathrm{nM}$ and used as standard. Serum was 10 -fold diluted for measurements. Receptor activation was calculated as chemerin-156 equivalents.

Immunoblotting. Immunoblotting was performed as described elsewhere (23). Antibodies for caveolin-1, fatty acid synthase (FAS), glyceraldehyde-3-phosphate dehydrogenase (GAPDH) and stearoylCoA desaturase 1 (SCD1) were from New England Biolabs GmbH (Frankfurt am Main, Germany). CMKLR1 antibody was from Novus Biologicals (Wiesbaden Nordenstadt, Germany). Sterol regulatory element-binding protein (SREBP) 1c antibody was from Thermo Fisher Scientific (Schwerte, Germany). SREBP2 antibody was from Cayman Chemicals (IBL International GmbH, Ann Arbor, MI, USA). Chemerin antibody was from R\&D Systems.

Sirius Red and hematoxylin-eosin staining. Liver tissues were fixed in $4 \%$ formalin solution and embedded in paraffin. Sections with a thickness of $3 \mu \mathrm{m}$ were prepared. After deparaffinization (Histol; Carl Roth, Karlsruhe, Germany) (two washes for $10 \mathrm{~min}$ each) and rehydration in ethanol with two washes for $5 \mathrm{~min}$ in $100 \%, 96 \%$ and $70 \%$ and one wash for $5 \mathrm{~min}$ in distilled water, the slides were incubated for $2 \mathrm{~min}$ in hematoxylin solution (Carl Roth). After washing with tap water for $10 \mathrm{~min}$, the slides were stained for $5 \mathrm{~min}$ with Eosin G solution (Carl Roth). Tissues were dehydrated by the sequential transfer through an increasing alcohol gradient (two washes for $2 \min$ in $70 \%, 96 \%$ and $100 \%$ ) and then incubated with Histol (twice for 5 min each). Entellan (Merck Chemicals GmbH, Darmstadt, Germany) served as mounting medium. Sirius Red (Direct Red 80; Sigma) staining was performed for 30 min using rehydrated tissues which were subsequently dehydrated as described above. Three different sections of each of the tissue slides were photographed and representative photographs are shown.

Real-time reverse transcription polymerase chain reaction ( $R T$ $P C R$ ). Real-time RT-PCR of chemerin and CMKLR1 was carried out as recently described and $18 \mathrm{~S}$ rRNA expression was analyzed as a housekeeper $(6,24)$.

Statistical analysis. Data are shown as box plots (median, lower and upper quartiles and range of the values). Small circles are used for outliers greater than 1.5 times the interquartile range and stars for outliers greater than 3.0 times the interquartile range. Statistical differences were analyzed by one-way ANOVA with post-hoc 
Bonferroni test, Mann-Whitney $U$-test (SPSS Statistics 19.0 program; IBM, Leibniz Rechenzentrum, Munich, Germany) and MS Excel for paired data and differences with values of $p<0.05$ were regarded as significant.

\section{Results}

DEN injection has no effect on body composition and liver steatosis. Animals fed the LMCD or the respective normal chow had similar body weights at the end of the study (Figure 1A). Injection of DEN had no effect on final body weight of either group (Figure 1A). Liver-to-body weight ratio increased in mice fed the LMCD diet irrespective of DEN treatment (Figure 1B). Subcutaneous and peri-renal adipose tissue weight was changed neither by diet nor by DEN injection (Figure 1C and data not shown). Epididymal fat mass significantly increased in control-injected mice fed the LMCD diet. Gain of fat mass was not significant in the DEN-injected mice fed the LMCD diet. The weight of the epididymal fat did not, however, differ between the two groups of mice fed the LMCD chow (Figure 1D). LMCD diet feeding caused liver steatosis (Figure 1E), the livers were bright and histological examination revealed the existence of numerous lipid droplets (Figure 1E).

DEN injection has no effect on proteins regulating lipid synthesis. MCD diet lowers SREBP1c, SCD1 and FAS expression in the liver $(25,26)$. Down-regulation of expression of these proteins was also detected in the LMCD model (Figure 2A-D). SREBP1c was also reduced in the DEN-injected mice fed the LMCD diet, while SCD1 and FAS only tended to be lower (Figure 2A-D). There was, however, no significant difference in the levels of these proteins when comparing water and DEN-injected mice fed an LMCD diet. Thus, injection of the carcinogen did not modulate the level of any of these proteins (Figure 2A-D). SCD1 was even lower in the liver of DEN-injected mice fed normal feed compared to control animals (Figure 2C). SREBP2 and caveolin-1 function in cholesterol homeostasis $(27,28)$. Protein expression of inactive full-length SREBP2 and proteolytic activated SREBP2 was down-regulated in the liver of mice fed the LMCD diet irrespective of carcinogen injection (Figure 2A and E, and data not shown). Caveolin1 expression was lower in the liver of water-injected mice fed an LMCD diet and tended to be reduced by the NASHinducing feed in DEN-treated animals (Figure 2F).

DEN injection has no effect on liver fibrosis. Sirius Red staining revealed development of liver fibrosis in mice given the LMCD diet. DEN treatment did not modulate extent of liver fibrosis (Figure 2G).

DEN injection has no effect on hepatic and total serum chemerin. Hepatic chemerin protein is induced in mice fed
MCD diet (6) and this was also the case in animals fed the LMCD diet compared with the control diet (Figure 3A and B). Chemerin mRNA expression tended to be higher in the liver of control water-injected mice fed the LMCD diet and was significantly induced in DEN-injected mice on the LMCD diet (Figure 3C). CMKLR1 protein was not dysregulated in the liver of LMCD-fed mice (Figure 3D and E). DEN did not affect CMKLR1 protein levels, while mRNA expression was strongly induced in the liver of DENtreated LMCD-fed mice compared with both respective controls (Figure 3D-F).

Total serum chemerin was higher in the control and DENinjected mice fed the LMCD diet compared to the respective control animals (Figure 3G). Serum chemerin bioactivity (chemerin-156 equivalents) as determined by ex vivo CMKLR1 and GPR1 activation was compared between DEN and control-injected mice fed the LMCD diet. While there was no difference in CMKLR1 activation relative to total chemerin protein level, GPR1 activation tended to be lower in serum of DEN-treated animals ( $p=0.114$ ) (Figure $3 \mathrm{H}$ and I).

DEN injection promotes tumor development in mice with $N A S H$. Liver tumors were not seen in mice injected with water as control irrespective of their diet. There were about two tumors per liver in the DEN-treated animals fed the control chow. When combined with LMCD diet, tumor number per liver increased (Figure 4A). In general, tumors were very small and did not exceed a diameter of $0.4 \mathrm{~mm}$ (data not shown). The level of serum adiponectin, which purportedly protects from NASH and liver cancer $(1,29)$, was decreased in DEN-injected mice fed the LMCD diet compared with DEN-injected mice fed the control chow (Figure 4B). Such a decline was not seen in the waterinjected animals (Figure 4B).

Chemerin mRNA levels were similar in tumor and nontumorous tissues of DEN-injected mice fed the control diet. There was a decline of chemerin mRNA in tumor of DENinjected mice fed the LMCD diet when compared to nontumorous tissues (Figure 4C). Chemerin protein was not changed in tumor compared to non-tumorous tissues $(p=0.07)$ (Figure 4D and E). CMKLR1 protein expression was not modified in tumor tissues, while the mRNA level was lower in tumor compared to non-tumorous tissues of mice fed the LMCD diet (Figure 4F and G).

\section{Discussion}

The present analyses demonstrated that serum chemerin protein and ex vivo activation of CMKLR1 and GPR1 were unchanged in NASH-related HCC when compared to NASH. Chemerin protein was increased in the liver in mice with $\mathrm{NASH}$, suggesting that carcinogenesis develops despite high levels of supposedly protective chemerin protein. 

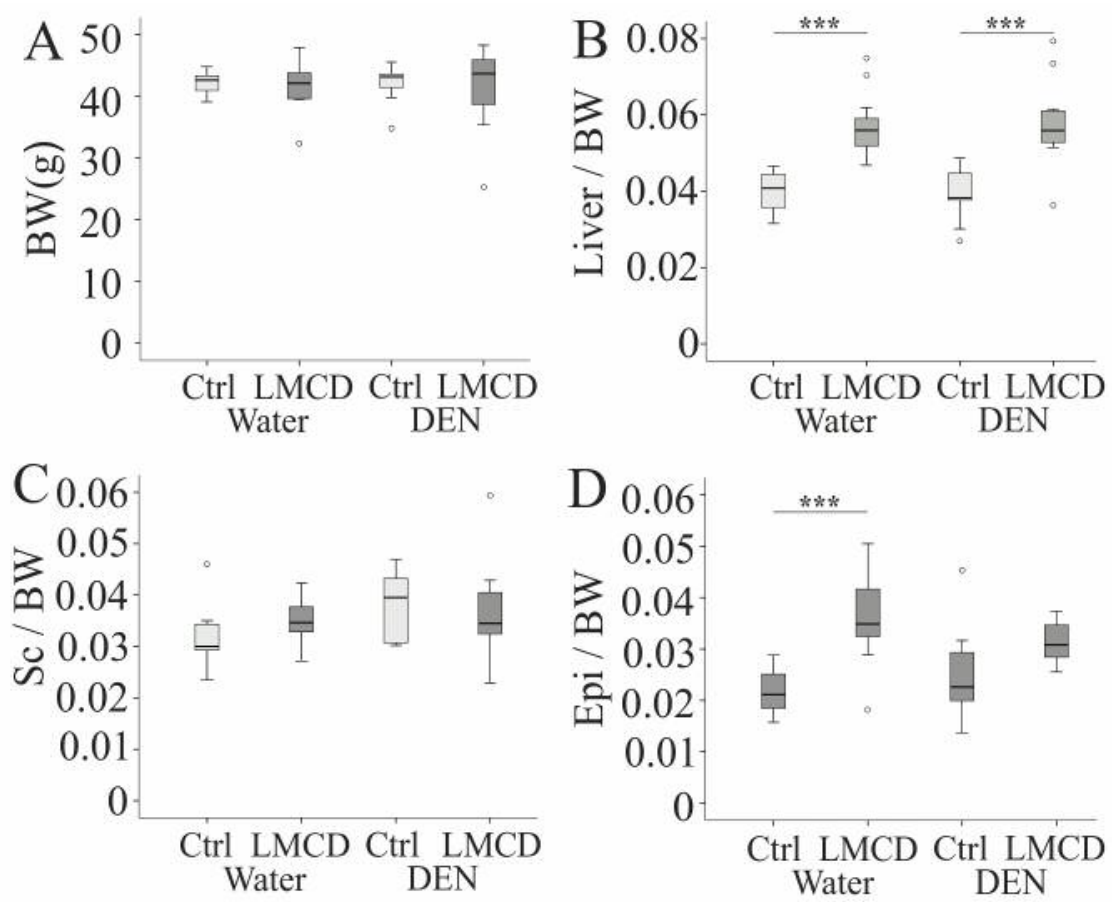

$\mathrm{E}$
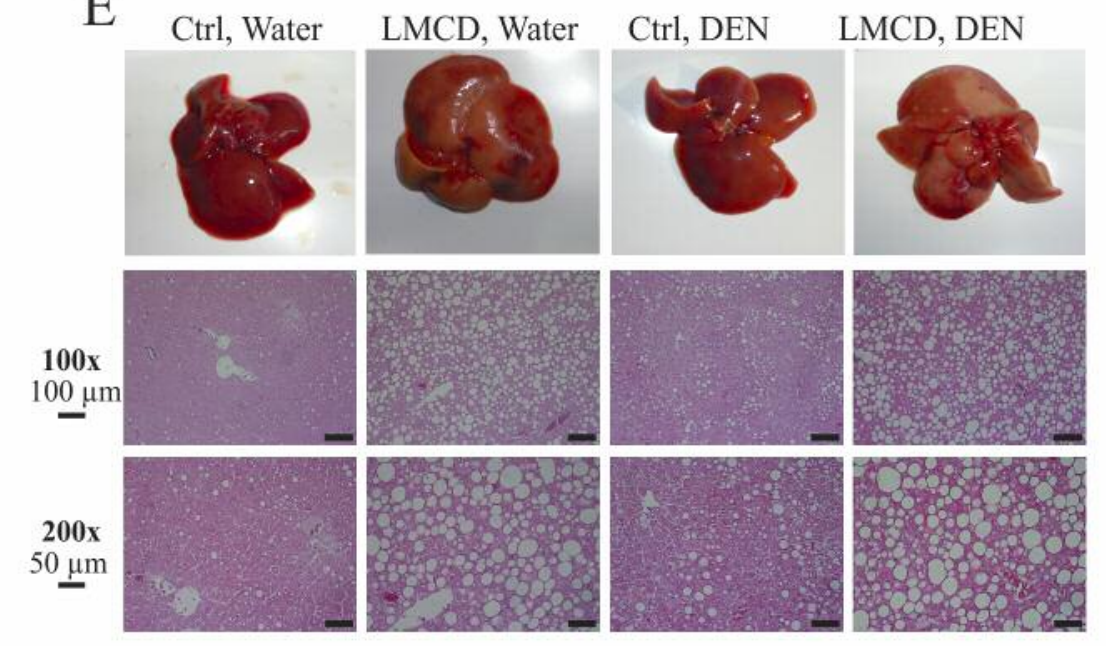

LMCD, DEN

Figure 1. Body weight $(B W)$, adipose tissue weights and hepatic histomorphological evaluation. Mice were fed a control (Ctrl) or low-methionine choline-deficient (LMCD) diet, and injected either with water as control or diethylnitrosamine (DEN) (n=10-11 mice per group). A: BW. B: Liverto- $B W$ ratio. $C$ : Relative subcutaneous adipose tissue $(S c)$ weight. D: Relative weight of epididymal adipose tissue (Epi). E: Appearance of the livers and liver tissues stained with hematoxylin-eosin. ***Significantly different at $p<0.001$.

A recent study of overexpression or injection of chemerin156 in an orthotopic mouse model of HCC nevertheless showed that chemerin protects from HCC (7). Chemerin-156 may therefore represent a low abundant hepatic chemerin isoform and might not be induced in liver in NASH.

Total chemerin protein analysis alone gives no information on the distribution of chemerin isoforms and its bioactivity. Several biologically active chemerin isoforms have been identified and they all derive from inactive, fulllength chemerin through cleavage of C-terminally located amino acids $(2,5)$. The composition of hepatic chemerin isoforms has not been analyzed and neither immunoblot nor ELISA techniques can distinguish between the different variants. Thus, it is possible that chemerin activity is changed in tumor tissues while the total chemerin protein level remains practically normal. Even a higher chemerin 

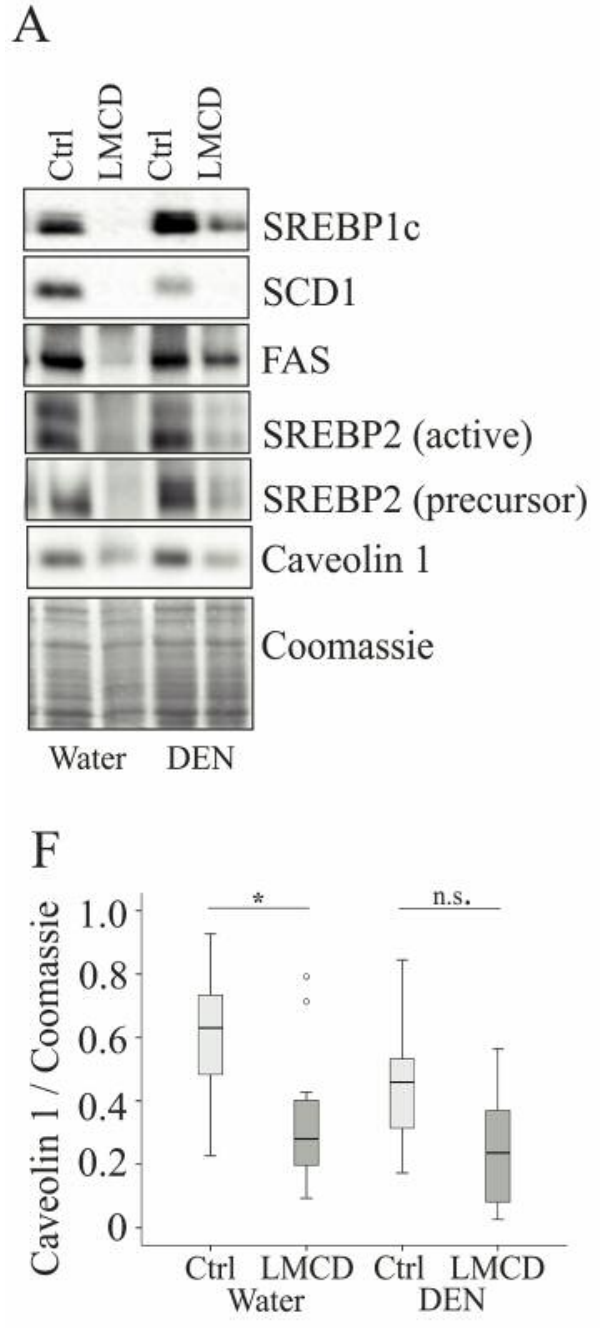
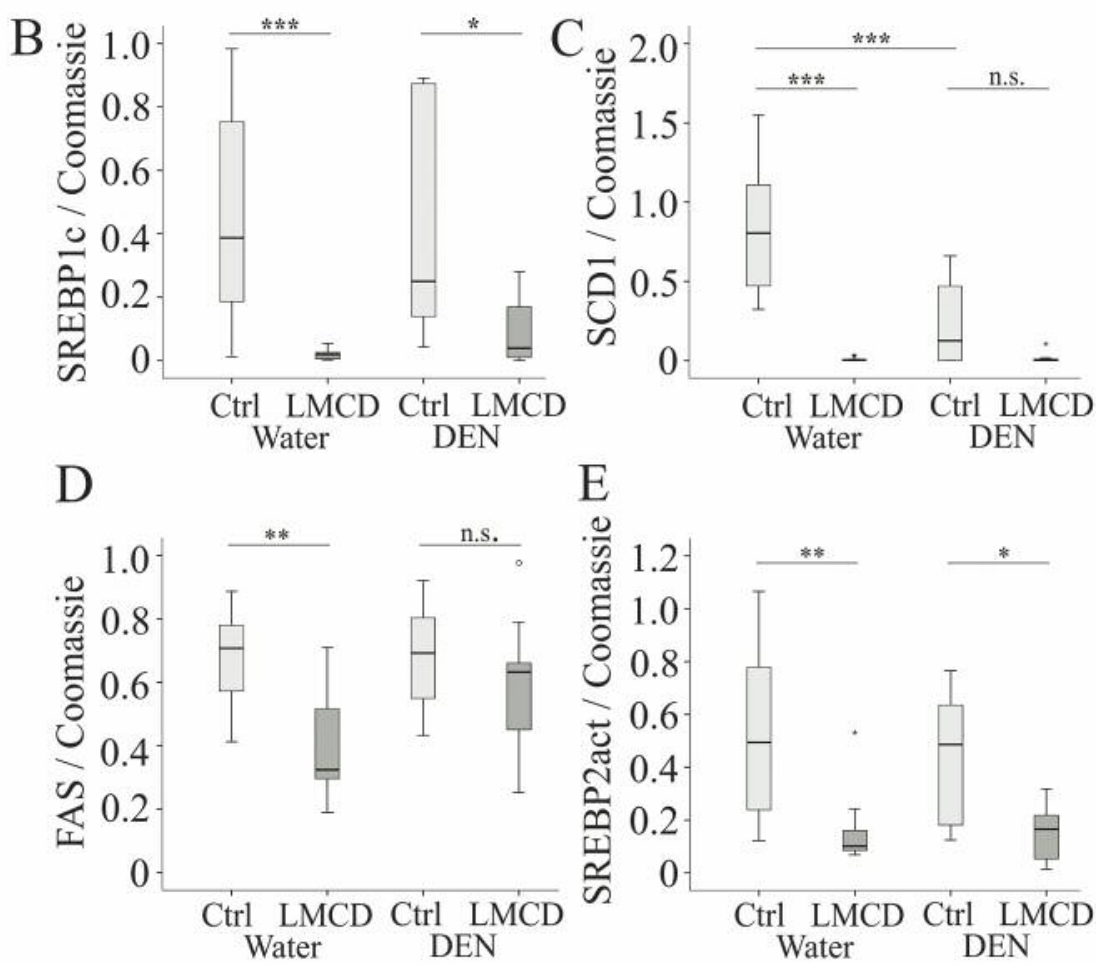

E
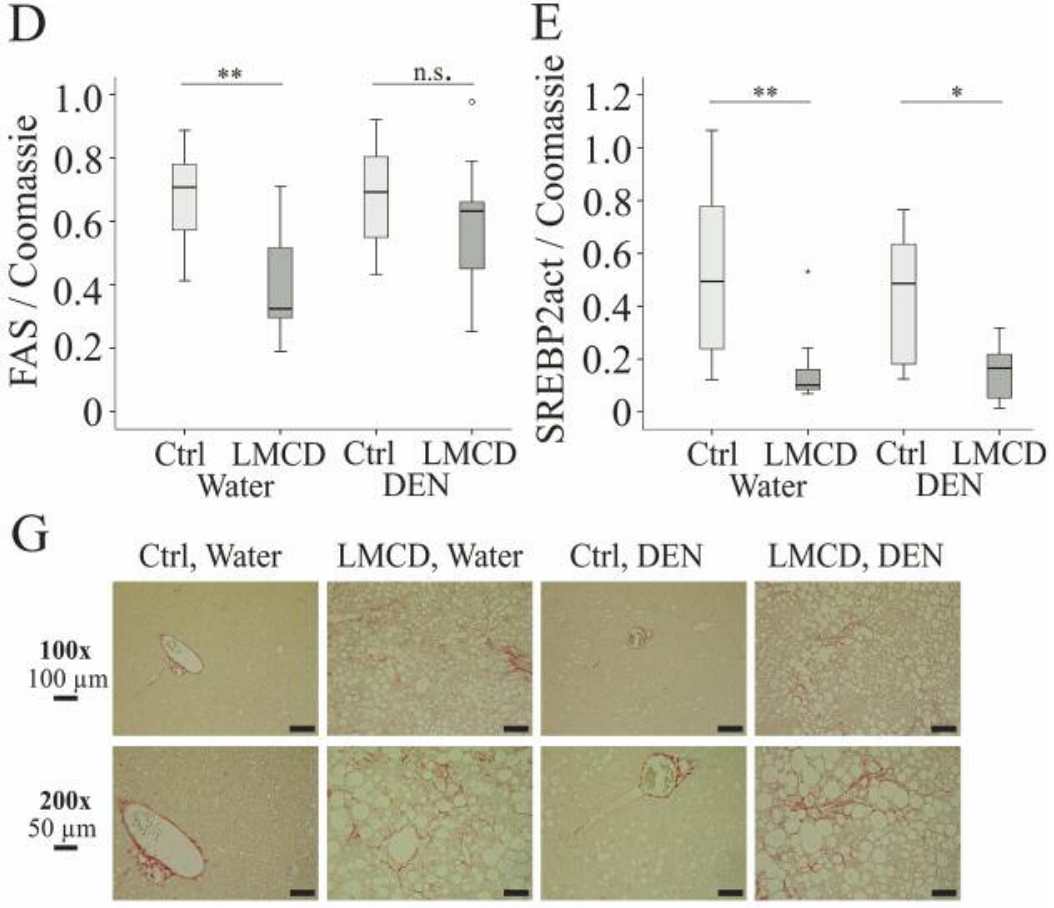

Figure 2. Hepatic protein expression and analysis of liver fibrosis in mice fed a control (Ctrl) or low-methionine choline-deficient (LMCD) diet, and injected either with water as control or diethylnitrosamine (DEN) (n=10-11 mice per group). A: Immunoblot of hepatic sterol regulatory elementbinding protein (SREBP) 1c, stearoyl-CoA desaturase 1 (SCD1), fatty acid synthase (FAS), SREBP2 (active and precursor form) and caveolin-1. Quantification of proteins: B: SREBP1c; C: SCD1; D: FAS; E: SREBP2; F: Caveolin-1. Coomassie-stained membranes served as loading controls. $G$ : Images of liver tissues stained with Sirius Red to detect collagen fibers. Significantly different at: $*_{p}<0.05, * * p<0.01, * * * p<0.001 . n . s$. Not significantly different $(p \geq 0.05)$.

protein level in liver in NASH does not predict increased hepatic chemerin activity.

CMKLR1 protein expression was found to be unchanged in liver in NASH and HCC tissues, excluding the possibility that an altered level of this receptor contributes to changed chemerin activity. Studies on CMKLR1 expression in murine and human NASH published so far reported normal, low and modestly induced levels $(8,30,31)$. Our finding that the CMKLR1 protein level was not changed is in accordance with previous findings in mice fed an atherogenic diet to induce NASH (31). GPR1 protein was finally not analyzed in the livers in our study because preliminary experiments failed to demonstrate the specificity of two different antibodies tested so far (data not shown).

Feeding an MCD diet is a common NASH model (19). Hepatic gene expression in such a model closely resembles human NASH (32), while body weight loss is regarded as a disadvantage of this model (19). By contrast, methioninerestricted, choline-deficient chows maintain body weight, and cause liver steatosis and fibrosis (20). This is also seen in the murine model used herein. Levels of SREBP1c, SREBP2, FAS, SCD1 and caveolin-1 protein, which are 
A

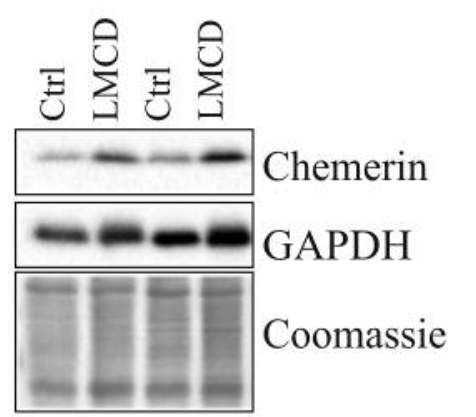

Water DEN

D

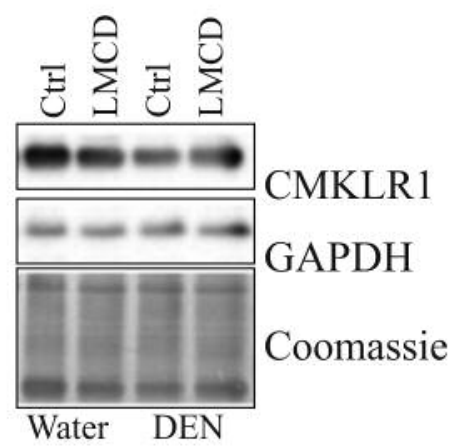

G

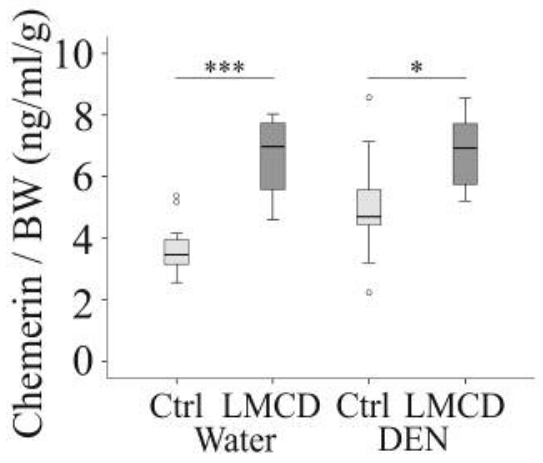

E
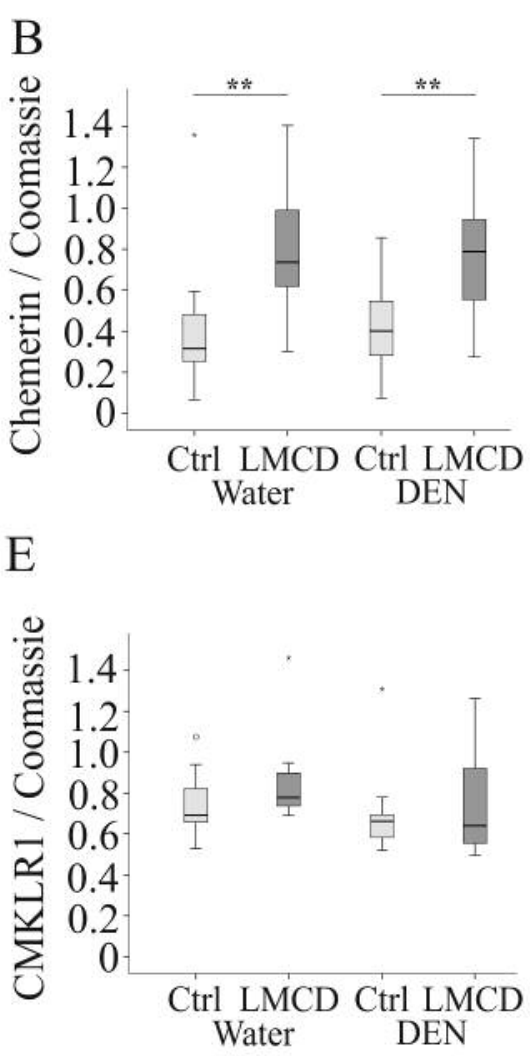

$\mathrm{H}$

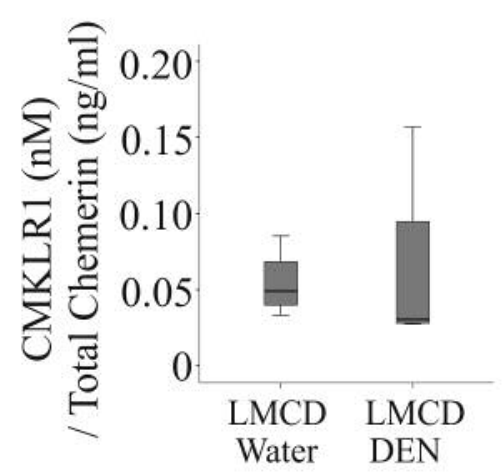

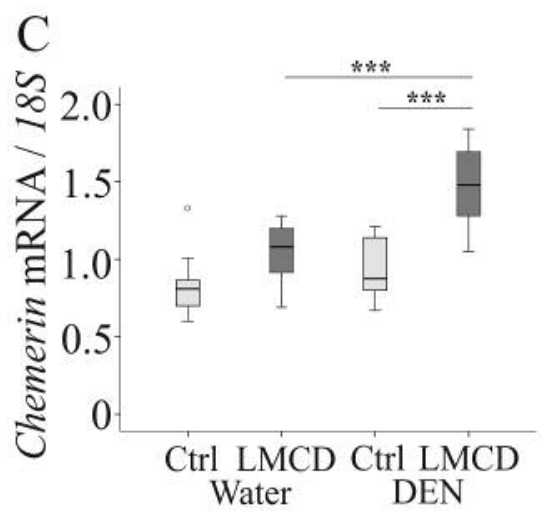

F
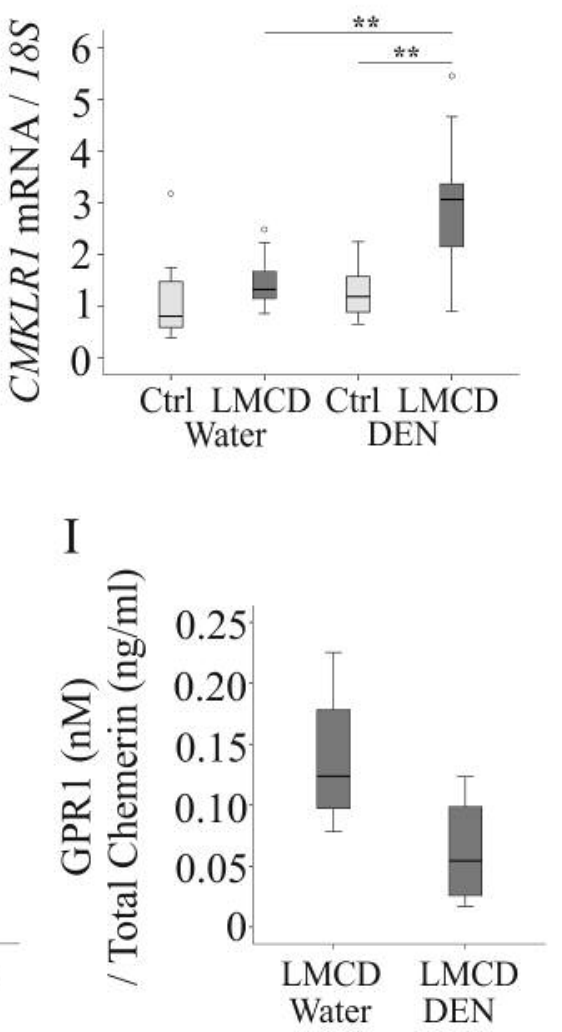

Figure 3. Analysis of hepatic and systemic chemerin and serum activation of chemokine-like receptor 1 (CMKLR1) and G-protein-coupled receptor 1 (GPR1) in mice fed a control (Ctrl) or low-methionine choline-deficient (LMCD) diet, and injected either with water as control or diethylnitrosamine (DEN) (n=10-11 mice per group). A: Immunoblot of hepatic chemerin and glyceraldehyde-3-phosphate dehydrogenase (GAPDH). Quantification of B: chemerin protein (Coomassie-stained membranes served as loading controls) and C: chemerin mRNA normalized to $18 S$ rRNA. D: Immunoblot of hepatic CMKLR1 and GAPDH. Quantification of E: CMKLR1 protein (Coomassie-stained membranes served as loading controls) and F: CMKLR1 mRNA normalized to $18 S$ rRNA. G: Total serum chemerin normalized to body weight (BW). Serum activation of H: CMKLR1 and I: GPR1 given as chemerin-156 equivalents and normalized to total serum chemerin in four mice injected with water and four mice injected with DEN which were all fed an LMCD diet. Significantly different at: *p $<0.05, * * p<0.01$ and $* * * p<0.001$.

involved in homeostasis of hepatic lipids, were reduced in the liver in NASH due to feeding the LMCD diet. SREBP1c, SCD1 and FAS levels were also lower in the liver in NASH of mice fed an MCD $(25,26)$. Caveolin-1 protects from hepatic lipid accumulation and is low in the liver of mice fed a high-fat diet (33). Caveolin-1 and SREBP2 have not been analyzed in liver from animals fed an MCD to our knowledge, but may be reduced similarly to mice fed the 


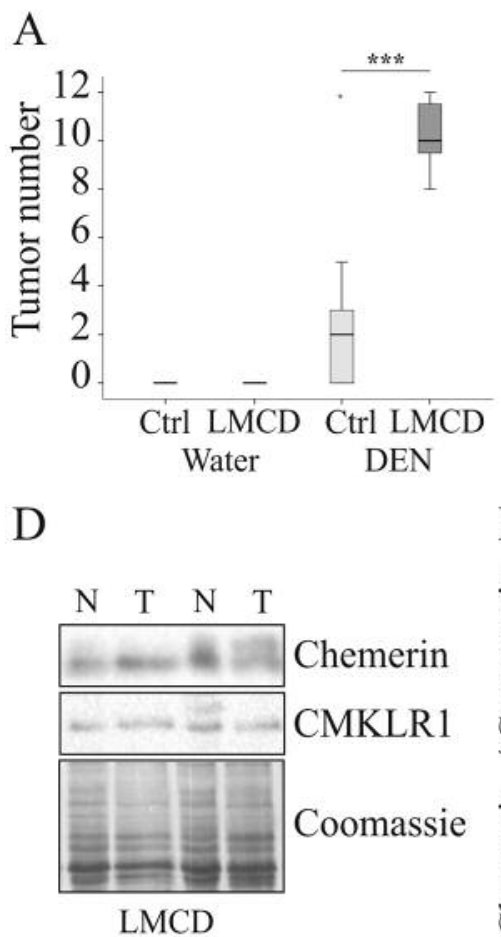

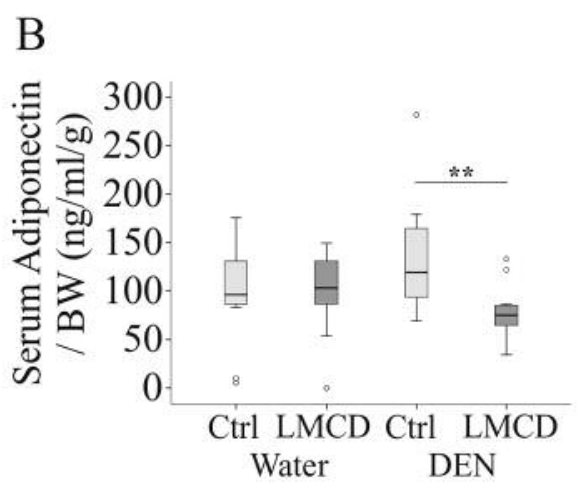

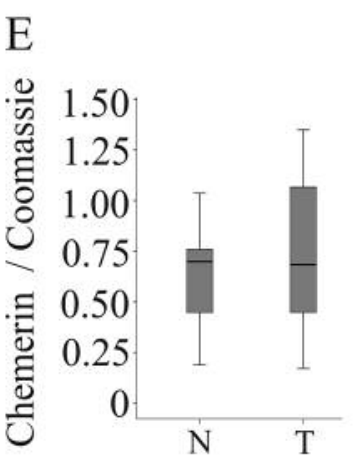

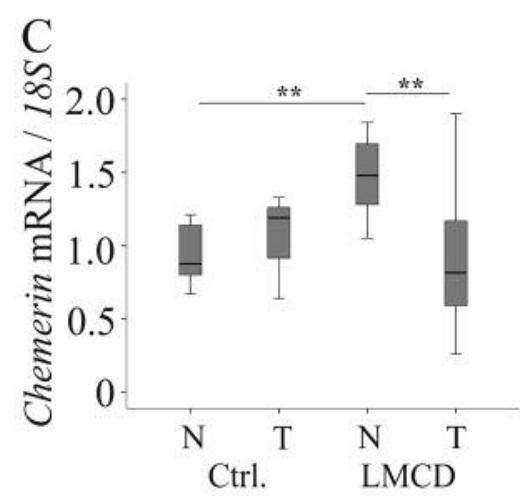

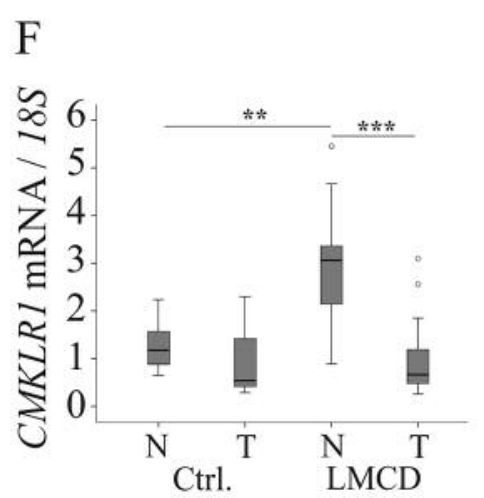

G

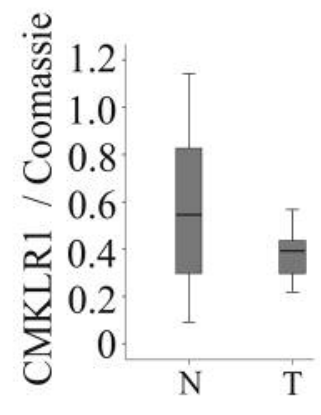

Figure 4. Number of tumors, serum adiponectin, and tumor chemerin and chemokine-like receptor 1 (CMKLR1) protein in mice fed a control (Ctrl) or a low-methionine choline-deficient (LMCD) diet, and injected either with water as control or diethylnitrosamine (DEN) ( $n=10-11$ mice per group). A: Number of tumors per liver. B: Serum adiponectin normalized to body weight (BW). C: Chemerin mRNA normalized to $18 S$ rRNA in tumorous $(T)$ and non-tumorous $(N)$ tissues of mice. D: Immunoblot of hepatic chemerin and CMKLR1 of tissue of mice injected with DEN and fed an LMCD diet. E: Quantification of chemerin protein in tissues of mice injected with DEN and fed an LMCD diet. F: CMKLR1 mRNA normalized to $18 S$ rRNA in tissues of DEN-injected mice fed a control or an LMCD diet. G: Quantification of CMKLR1 in tissue of mice injected with DEN and fed an LMCD diet. Coomassie-stained membranes served as loading controls. Significantly different at: ** $p<0.01, * * * p<0.001$.

LMCD diet. Present analysis shows that fatty acid and cholesterol synthesis are markedly suppressed in the liver of mice fed an LMCD diet. Thus, the described impaired release of hepatic lipoprotein particles caused by choline deficiency contributes to lipid accumulation in the liver (34) even when endogenous production of lipids is suppressed.

SCD1 was suppressed in the liver of DEN-injected mice when compared to control-injected animals fed a control diet. Most studies compare SCD1 levels in non-tumorous and tumor tissue, and SCD1 was found to be induced in HCC (35). We are unaware of studies comparing SCD1 in normal liver tissues of patients with HCC and controls. SCD1 contributes to tumorigenesis (36) and its downregulation may thereby be protective.

Hepatic carcinogenesis was found not to change the levels of the other proteins specified above, nor lipid droplet size or the degree of fat deposition in the liver. Hepatic fibrosis assessed by staining of collagen fibers with Sirius Red was detected in mice fed the LMCD diet irrespective of tumor load.
Hepatic chemerin protein was increased in the liver in NASH. Higher levels have been described in mice fed the MCD diet and mice given an atherogenic chow to induce NASH (6). It is important to note that chemerin expression declined in rats fed an MCD diet (9). Discordant findings in mice and rats may reflect species-specific differences and future research should resolve this issue. In human studies, to our knowledge, only hepatic chemerin mRNA expression has been determined $(8,11)$. Because mRNA levels may not reflect hepatic protein content, analysis of protein is essential in order to further evaluate chemerin levels in liver in human NASH $(8,11)$. Most importantly, the distribution of chemerin isoforms and their physiological activities need to be studied to further understand the role of chemerin in NASH and HCC.

Of note, although body and fat mass were not grossly induced in the LMCD-fed groups, total chemerin protein in the serum was increased. Chemerin may be released by the liver and adipose tissues in NASH, and the latter may mostly contribute to serum chemerin level $(2,5)$. Analysis of 
adipose tissue chemerin production in MCD and LMCD models may help to identify the source of increased circulating chemerin in these animals. Importantly, serum chemerin and ex vivo activation of CMKLR1 and GPR 1 were unchanged in murine HCC. There was nevertheless a trend towards decreased bioactivation of GPR1, suggesting modestly impaired chemerin signaling by the GPR $1-\beta$ arrestin 2 pathway in HCC. The pathophysiologic role of this pathway in HCC development has, however, not been analyzed to our knowledge. A limitation of the activity assay used in the present study is that only $\beta$-arrestin 2 -dependent signaling was monitored. Accumulating evidence indicates that CMKLR1 and GPR1 communicate through multiple cell signaling pathways $(15,37)$, which should be studied in detail to define the role of chemerin bioactivity in NASH and NASH-associated HCC.

The level of adiponectin was determined in the serum of these animals, and a decline was only seen in DEN-injected mice fed the LMCD diet. Adiponectin deficiency contributes to the pathogenesis of HCC (38) and may augment tumor progression in the mouse model used herein.

The present study showed that chemerin mRNA and protein are not always coordinately changed; this also applies to CMKLR1. Thus, mRNA expression of both was induced in the liver of DEN-injected mice fed an LMCD diet, while the respective protein levels were unchanged: in tumor from these mice, the mRNA levels were reduced when compared to the respective normal tissue, and again, the proteins levels were unchanged. Although the underlying mechanisms involved therein are unknown, these data suggest dysregulation of chemerin and CMKLR1 mRNA or protein synthesis/stability in DEN-treated mice on a NASH diet and in tumor tissue.

The present study showed that the increase of hepatic and systemic chemerin in murine NASH are both unchanged upon tumorigenesis. Endogenous chemerin protein seems not to protect from HCC, at least in the NASH-DEN model used herein.

\section{Acknowledgements}

The study was supported by grants from the Deutsche Forschungsgemeinschaft (BU 1141/13-1, CB) and the Canadian Institutes of Health Research (CJS).

\section{References}

1 Buechler C, Wanninger J and Neumeier M: Adiponectin, a key adipokine in obesity related liver diseases. World J Gastroenterol 17: 2801-2811, 2011.

2 Rourke JL, Dranse HJ and Sinal CJ: Towards an integrative approach to understanding the role of chemerin in human health and disease. Obes Rev 14: 245-262, 2013.

3 Buechler C: Chemerin in liver diseases. Endocrinol Metabol Syndr 3: 1-6, 2014.
4 Massoud O and Charlton M: Nonalcoholic fatty liver disease/ nonalcoholic steatohepatitis and hepatocellular carcinoma. Clin Liver Dis 22: 201-211, 2018.

5 Ernst MC and Sinal CJ: Chemerin: at the crossroads of inflammation and obesity. Trends Endocrinol Metab 21: 660-667, 2010.

6 Krautbauer S, Wanninger J, Eisinger K, Hader Y, Beck M, Kopp A, Schmid A, Weiss TS, Dorn C and Buechler C: Chemerin is highly expressed in hepatocytes and is induced in non-alcoholic steatohepatitis liver. Exp Mol Pathol 95: 199205, 2013.

7 Lin Y, Yang X, Liu W, Li B, Yin W, Shi Y and He R: Chemerin has a protective role in hepatocellular carcinoma by inhibiting the expression of IL-6 and GM-CSF and MDSC accumulation. Oncogene 36: 3599-3608, 2017.

8 Docke S, Lock JF, Birkenfeld AL, Hoppe S, Lieske S, Rieger A, Raschzok N, Sauer IM, Florian S, Osterhoff MA, Heller R, Herrmann K, Lindenmuller S, Horn P, Bauer M, Weickert MO, Neuhaus P, Stockmann M, Mohlig M, Pfeiffer AF and von Loeffelholz C: Elevated hepatic chemerin gene expression in progressed human non-alcoholic fatty liver disease. Eur J Endocrinol 169: 547-557, 2013.

9 Deng Y, Wang H, Lu Y, Liu S, Zhang Q, Huang J, Zhu R, Yang J, Zhang R, Zhang D, Shen W, Ning $G$ and Yang $Y$ : Identification of chemerin as a novel FXR target gene downregulated in the progression of nonalcoholic steatohepatitis. Endocrinology 154: 1794-1801, 2013.

10 Kajor M, Kukla M, Waluga M, Liszka L, Dyaczynski M, Kowalski G, Zadlo D, Berdowska A, Chapula M, KostrzabZdebel A, Buldak RJ, Sawczyn T and Hartleb M: Hepatic chemerin mRNA in morbidly obese patients with nonalcoholic fatty liver disease. Pol J Pathol 68: 117-127, 2017.

11 Pohl R, Haberl EM, Rein-Fischboeck L, Zimny S, Neumann M, Aslanidis C, Schacherer D, Krautbauer S, Eisinger K, Weiss TS and Buechler C: Hepatic chemerin mRNA expression is reduced in human nonalcoholic steatohepatitis. Eur J Clin Invest 47: 7$18,2017$.

12 Lin W, Chen YL, Jiang L and Chen JK: Reduced expression of chemerin is associated with a poor prognosis and a lowed infiltration of both dendritic cells and natural killer cells in human hepatocellular carcinoma. Clin Lab 57: 879-885, 2011.

13 Imai K, Takai K, Hanai T, Shiraki M, Suzuki Y, Hayashi H, Naiki T, Nishigaki Y, Tomita E, Shimizu M and Moriwaki H: Impact of serum chemerin levels on liver functional reserves and platelet counts in patients with hepatocellular carcinoma. Int J Mol Sci 15: 11294-11306, 2014.

14 Eisinger K, Krautbauer S, Wiest R, Weiss TS and Buechler C: Reduced serum chemerin in patients with more severe liver cirrhosis. Exp Mol Pathol 98: 208-213, 2015.

15 De Henau O, Degroot GN, Imbault V, Robert V, De Poorter C, McHeik S, Gales C, Parmentier M and Springael JY: Signaling properties of chemerin receptors CMKLR1, GPR1 and CCRL2. PLoS One 11: e0164179, 2016.

16 Toulany J, Parlee SD, Sinal CJ, Slayter K, McNeil S and Goralski KB: CMKLR1 activation ex vivo does not increase proportionally to serum total chemerin in obese humans. Endocr Connect 5: 70-81, 2016.

17 Haberl EM, Pohl R, Rein-Fischboeck L, Feder S, Eisinger K, Krautbauer S, Sinal CJ and Buechler C: Ex vivo analysis of serum chemerin activity in murine models of obesity. Cytokine 104: 42-45, 2018. 
18 Takizawa D, Kakizaki S, Horiguchi N, Yamazaki Y, Tojima H and Mori M: Constitutive active/androstane receptor promotes hepatocarcinogenesis in a mouse model of non-alcoholic steatohepatitis. Carcinogenesis 32: 576-583, 2011.

19 Schattenberg JM and Galle PR: Animal models of non-alcoholic steatohepatitis: of mice and man. Dig Dis 28: 247-254, 2010.

20 Matsumoto M, Hada N, Sakamaki Y, Uno A, Shiga T, Tanaka C, Ito T, Katsume A and Sudoh M: An improved mouse model that rapidly develops fibrosis in non-alcoholic steatohepatitis. Int $\mathbf{J}$ Exp Pathol 94: 93-103, 2013.

21 Toriguchi K, Hatano E, Tanabe K, Takemoto K, Nakamura K, Koyama Y, Seo S, Taura K and Uemoto S: Attenuation of steatohepatitis, fibrosis, and carcinogenesis in mice fed a methionine-choline deficient diet by CCAAT/enhancer-binding protein homologous protein deficiency. J Gastroenterol Hepatol 29: 1109-1118, 2014

22 Rourke JL, Muruganandan S, Dranse HJ, McMullen NM and Sinal CJ: Gpr1 is an active chemerin receptor influencing glucose homeostasis in obese mice. J Endocrinol 222: 201-215, 2014.

23 Krautbauer S, Eisinger K, Hader Y and Buechler C: Free fatty acids and IL- 6 induce adipocyte galectin- 3 which is increased in white and brown adipose tissues of obese mice. Cytokine 69: 263-271, 2014.

24 Wanninger J, Bauer S, Eisinger K, Weiss TS, Walter R, Hellerbrand C, Schaffler A, Higuchi A, Walsh K and Buechler $\mathrm{C}$ : Adiponectin upregulates hepatocyte CMKLR1 which is reduced in human fatty liver. Mol Cell Endocrinol 349: 248-254, 2012.

25 Rizki G, Arnaboldi L, Gabrielli B, Yan J, Lee GS, Ng RK, Turner SM, Badger TM, Pitas RE and Maher JJ: Mice fed a lipogenic methionine-choline-deficient diet develop hypermetabolism coincident with hepatic suppression of SCD1. J Lipid Res 47: 2280-2290, 2006.

26 Jha P, Knopf A, Koefeler H, Mueller M, Lackner C, Hoefler G, Claudel $\mathrm{T}$ and Trauner M: Role of adipose tissue in methioninecholine-deficient model of non-alcoholic steatohepatitis (NASH). Biochim Biophys Acta 1842: 959-970, 2014.

$27 \mathrm{Gu}$ HM, Wang FQ and Zhang DW: Caveolin-1 interacts with ATP binding cassette transporter G1 (ABCG1) and regulates ABCG1-mediated cholesterol efflux. Biochim Biophys Acta 1841: 847-858, 2014.

28 Musso G, Gambino R and Cassader M: Cholesterol metabolism and the pathogenesis of non-alcoholic steatohepatitis. Prog Lipid Res 52: 175-191, 2013.
29 Wieser V, Moschen AR and Tilg H: Adipocytokines and hepatocellular carcinoma. Dig Dis 30: 508-513, 2012.

30 Neumann M, Meier EM, Rein-Fischboeck L, Krautbauer S, Eisinger K, Aslanidis C, Pohl R, Weiss TS and Buechler C: Chemokine-like receptor 1 mRNA weakly correlates with nonalcoholic steatohepatitis score in male but not female individuals. Int J Mol Sci 17: 1335, 2016.

31 Pohl R, Rein-Fischboeck L, Meier EM, Eisinger K, Krautbauer $\mathrm{S}$ and Buechler C: Resolvin E1 and chemerin C15 peptide do not improve rodent non-alcoholic steatohepatitis. Exp Mol Pathol 98: 295-299, 2015.

32 Teufel A, Itzel T, Erhart W, Brosch M, Wang XY, Kim YO, von Schonfels W, Herrmann A, Bruckner S, Stickel F, Dufour JF, Chavakis T, Hellerbrand C, Spang R, Maass T, Becker T, Schreiber S, Schafmayer C, Schuppan D and Hampe J: Comparison of gene expression patterns between mouse models of nonalcoholic fatty liver disease and liver tissues from patients. Gastroenterology 151: 513-525 e510, 2016.

33 Li M, Chen D, Huang H, Wang J, Wan X, Xu C, Li C, Ma H, $\mathrm{Yu} \mathrm{C}$ and $\mathrm{Li} \mathrm{Y}$ : Caveolin 1 protects against diet induced hepatic lipid accumulation in mice. PLoS One 12: e0178748, 2017.

34 Rinella ME, Elias MS, Smolak RR, Fu T, Borensztajn J and Green RM: Mechanisms of hepatic steatosis in mice fed a lipogenic methionine choline-deficient diet. J Lipid Res 49: 1068-1076, 2008.

35 Muir K, Hazim A, He Y, Peyressatre M, Kim DY, Song X and Beretta L: Proteomic and lipidomic signatures of lipid metabolism in NASH-associated hepatocellular carcinoma. Cancer Res 73: 4722-4731, 2013.

36 Ma MKF, Lau EYT, Leung DHW, Lo J, Ho NPY, Cheng LKW, Ma S, Lin CH, Copland JA, Ding J, Lo RCL, Ng IOL and Lee TKW: Stearoyl-CoA desaturase regulates sorafenib resistance via modulation of ER stress-induced differentiation. J Hepatol 67: 979-990, 2017.

37 Rourke JL, Dranse HJ and Sinal CJ: CMKLR1 and GPR1 mediate chemerin signaling through the RhoA/ROCK pathway. Mol Cell Endocrinol 417: 36-51, 2015.

38 Adolph TE, Grander C, Grabherr F and Tilg H: Adipokines and non-alcoholic fatty liver disease: Multiple Interactions. Int J Mol Sci 18: 1649, 2017.

Received March 7, 2018

Revised March 26, 2018

Accepted April 2, 2018 